\title{
Envejecimiento activo y saludable: investigación y políticas para el envejecimiento poblacional
}

\section{Active and healthy aging. Research and policies for population aging}

Por estos días comenzamos a conocer los primeros resultados del Censo 2012. Tenemos entonces una nueva oportunidad para reflexionar sobre esta relación circular entre población y salud que ha determinado cambios abismales en períodos relativamente reducidos. La caída abrupta de la mortalidad es sin duda un logro mayor, la cual sumada a la caída de la fecundidad interrumpieron siglos de constantes demográficas, configurando transiciones en todos los sectores, con sus inevitables consecuencias sobre la salud poblacional.

El aumento de la dependencia es el costo inevitable de estos cambios demográficos. La población mundial de 60 años o más supera los 650 millones y se calcula que en 2050 alcanzará a 2000 millones. Proporcionalmente aumenta más rápidamente que cualquier otro grupo de edad en casi todos los países, proceso que naturalmente, va más adelantado en los países desarrollados. En nuestro país la proporción estimada de mayores de 60 años en el año 2000 era 10\%; en el año 2010 ya era de 13\% y se anticipa que será de $17 \%$ en el año 2020. En términos absolutos, serán 3.207 .729 personas, vale decir que ese grupo se duplicará en 20 años ${ }^{1}$.

Si bien el envejecimiento poblacional es un indicador del éxito de las políticas de salud pública y del desarrollo socioeconómico, constituye al mismo tiempo un gran desafio para la sociedad. Esta no sólo debe lidiar con la nueva situación optimizando la salud y la capacidad funcional de los adultos mayores, sino que debe lograr su integración y participación social, apuntando en última instancia a su calidad de vida.

En términos de la optimización de la salud, la transición epidemiológica ha implicado enormes desafios, comenzando por la mayor expresión de las enfermedades crónicas respiratorias, cardiovasculares y neoplásicas. Como antes ocurrió con las enfermedades transmisibles, paulatinamente se han ido develando los factores de riesgo, lo que ha permitido modular el ritmo de aumento de su incidencia, en tanto que notables avances terapéuticos y quirúrgicos han logrado disminuir su letalidad.

En el ámbito especifico de la salud respiratoria se destaca que, pese a los múltiples cambios desfavorables que sufre el sistema respiratorio con la senescencia, el pulmón de las personas que han llevado una vida saludable y activa es capaz de mantener una ventilación y un intercambio gaseoso alvéolo-capilar aceptable, incluso más allá de los cien años de edad, lo que refleja la enorme reserva funcional del pulmón como órgano intercambiador de gases.2. Sin embargo, el condicionante de "vida saludable y activa" deja un espacio importante para la prevención, en la cual el médico tratante debe tener protagonismo.

Naturalmente la primera acción preventiva en el área se relaciona con el tabaquismo y, aunque en menor grado, con la exposición a contaminantes sean estos laborales, intradomiciliarios o atmosféricos. Si tales agresiones no son evitadas en etapas tempranas, la pendiente de declinación espirométrica será mayor a la que se produce por efecto de la edad, lo que sumado a la disminución de la depuración mucociliar, a la pérdida de la fuerza de los músculos inspiratorios y a la rigidez torácica-todos factores que ocurren con el envejecimiento-aumentará la vulnerabilidad de los adultos mayores. Entre tales factores de susceptibilidad, al menos la pérdida de fuerza muscular y la rigidez torácica-resultante de fracturas costales y vertebrales evidenciadas tardiamente en exámenes radiológicos del tórax-podrían ser contenidas evitando la vida sedentaria y previniendo la osteoporosis ${ }^{3}$.

En esta cadena de transiciones los avances preventivos y terapéuticos han llevado a una nueva etapa, tal vez la más cruel e ignota: las demencias. Frente a ellas, hasta ahora no se cuenta con claves para su prevención y los avances terapéuticos son mínimos, lo que conlleva la inmensa carga social de 
una sobrevivencia que demanda mayor asistencia. Por ahora ésta recae mayormente en las familias, lo que aumenta las exigencias de la sociedad moderna y podrá redundar en graves problemas sociales.

A fin de mejorar la calidad de vida de los adultos mayores y de evitar la problemática social asociada, el envejecimiento poblacional exige la concurrencia de políticas públicas que aminoren sus consecuencias en diversos aspectos de la vida: la familia, la economía, los servicios de salud y el entorno físico y comunitario ${ }^{4}$. El envejecimiento exige a las sociedades alterar el ciclo de trabajo y la oportunidad de la jubilación de sus miembros; obliga a las instituciones sociales a destinar recursos para la capacitación de los proveedores de servicios sociales y de atención de salud, de manera que atiendan eficaz y eficientemente a los adultos mayores. Plantea también requerimientos a los gobiernos centrales y locales para mejorar la infraestructura física que permita un medioambiente amigable y generar y apoyar actividades colectivas dirigidas a este grupo.

El estudio de los efectos económicos del envejecimiento poblacional debe concitar un interés especial. El análisis más simple de los efectos económicos de la transición demográfica comienza con la noción de dependencia basada en la edad: menores de 15 y mayores de 60 años, los cuales producen menos de lo que consumen y son dependientes de la población en edad laboral ${ }^{5}$. Sin embargo, en ese análisis se presenta una "ventana de oportunidades" durante la cual ocurre una reducción del número de personas dependientes, fundamentalmente menores de 15 años. La disminución debe dar como resultado el incremento del ingreso per-cápita de la población, permitiendo el incremento de los niveles de ahorro para la vejez o el nivel de transferencia por persona dependiente, al mismo tiempo que es posible mantener constante el ingreso disponible por persona en edad laboral. El aprovechamiento de esta ventana supone acciones prácticamente inmediatas pues ella se cerrará en menos de dos décadas.

El punto es que las tasas de dependencia cambian rápidamente. El riesgo está en que las poblaciones de los países en desarrollo envejezcan antes de alcanzar la riqueza, a diferencia de los países industrializados que se hicieron más ricos al tiempo que su población envejecía.

Las respuestas a esos desafios imponen la formidable tarea de producir el conocimiento requerido, tarea en la que las universidades tienen un papel crítico y que no debe limitarse al ámbito de la salud. Como se ve, hay desafios tanto o más complejos en lo referente al papel social en la exacerbación de los riesgos que enfrentan los adultos mayores.

En efecto, investigaciones recientes han mostrado la importancia de relaciones en dominios cruzados entre trabajo, retiro laboral, estatus económico, estructura familiar, salud y bienestar en edades avanzadas, temas para los cuales la investigación transdisciplinaria es imperativa. Temas que requieren también un avance desde metodologías transversales a longitudinales, lo que ya es posible a partir de seguimientos de grandes cohortes poblacionales. Por último, se debe hacer un esfuerzo para evaluar el papel definitorio de las políticas relacionadas al envejecimiento y el impacto de potenciales modificaciones a los programas existentes.

En este escenario, el sistema universitario y los centros de investigación de los países desarrollados han definido una amplia agenda de investigación que incorpora áreas tales como las siguientes: a) adultos mayores y su desarrollo: salud y envejecimiento saludable, sistema de seguridad social b) salud y el bienestar en la vejez: envejecimiento saludable, intervenciones biomédicas y funcionamiento físico y mental de los adultos mayores; mejoramiento de la calidad de vida y participación e integración social de los miembros de ese grupo c) medio ambiente: sistema de cuidado de la salud, estructuras y funciones de la familia; comunidades y ciudades amigables. En todas las áreas mencionadas, la evidencia producida por la investigación se utilizará para formular políticas y programas públicos.

Nuestro país no debe limitarse a esperar pasivamente los resultados de tal esfuerzo. El mundo académico formado por las universidades y las sociedades cientificas tienen la obligación de establecer alianzas para identificar y especificar los problemas relativos al envejecimiento que nuestra sociedad -con sus particulares características- enfrentará a futuro, de manera de propiciar el desarrollo de conocimientos y estrategias adecuadas para minimizarlos. Recientemente la Comisión Nacional de Investigación Científica y Tecnológica (Conicyt) ha incluido al envejecimiento entre las áreas prioritarias 
del Fondo de Financiamiento de Centros de Excelencia en Investigación (Fondap), lo que permitirá financiar centros de investigación avanzada y de formación de posgrado en esta temática. Otro hito trascendente promete ser la iniciativa de organizar una reunión internacional impulsada por el Dr. Jorge Allende. El destacado investigador, premio nacional de ciencias naturales, ha anunciado que el Consejo Nacional de Innovación para la Competitividad y la Facultad de Medicina de la Universidad de Chile están organizando dos talleres internacionales sobre este tema entre el 14 y 17 de enero de 2013, en los que participarán líderes mundiales en temas sociales, urbanísticos, económicos y de salud para compartir con sus colegas chilenos sus experiencias y resultados ${ }^{6}$.

La interdisciplinaridad deberá estar en el centro de este esfuerzo, inspirando a los investigadores de todas las áreas a considerar soluciones integradoras que involucren a la sociedad como un todo. No se trata sólo de buscar y encontrar soluciones para los problemas que enfrenta el grupo de los adultos mayores sino para la sociedad como un todo que envejece.

\section{José Sulbrandt C. ***, Paulina Pino Z.*** y Manuel Oyarzún G. ** * Escuela de Salud Pública. **Facultad de Medicina, Universidad de Chile.}

\section{Bibliografía}

1.- INE-CELADE 2008 CHILE: Proyecciones y Estimaciones de Población. Total País 1950-2050. Serie de la Publicación (CEPAL): OI N²08.

2.- OYARZÚN M. Función respiratoria en la senectud. Rev Méd Chile 2009; 137: 411-8.

3.- JANSSENS J P, PACHE J C, NICOD L P. Physiological changes in respiratory function associated with aging. Eur Respir J 1999; 13: 197-205.
4.- PELÁEZ M. La construcción de las bases de la buena salud en la vejez: situación en las Américas. Rev Panam Salud Pública 2005; 17: 299-302.

5.- SUÁREZ R, PESCETTO C. Sistemas de protección social para el adulto mayor en América Latina y el Caribe. Rev Panam Salud Pública 2005; 17: 419-28.

6.- ALLENDE J E. Envejecimiento poblacional. www.med. uchile.cl Consulta: 27.12.2012. 